Communications in Physics, Vol. 31, No. 2 (2021), pp. 199-2010

DOI:10.15625/0868-3166/15520

\title{
MULTIBAND METAMATERIAL ABSORBER BASE ON HIGH-ORDER MAGNETIC RESONANCE IN A RING STRUCTURE
}

\author{
NGUYEN THI HIEN ${ }^{1, \star}$, NGUYEN THI ANH HONG ${ }^{1}$, BUI XUAN KHUYEN ${ }^{2, \dagger}$, \\ BUI SON TUNG ${ }^{2, \ddagger}$, NGUYEN XUAN CA ${ }^{1}$, NGUYEN VAN NGOC ${ }^{3}$, \\ NGUYEN BA TUONG ${ }^{3}$ AND VU DINH LAM ${ }^{3}$ \\ ${ }^{1}$ Faculty of Physics and Technology, TNU-University of Sciences \\ Tan Thinh Ward, Thai Nguyen City, Thai Nguyen Province, Vietnam \\ ${ }^{2}$ Institute of Materials Science, Vietnam Academy of Science and Technology, \\ 18 Hoang Quoc Viet, Cau Giay, Hanoi, Vietnam \\ ${ }^{3}$ Graduate University of Science and Technology, Vietnam Academy of Science and Technology \\ 18 Hoang Quoc Viet, Cau Giay, Hanoi, Vietnam \\ E-mail: `hiennt@tnus.edu.vn; † khuyenbx@ims.vast.ac.vn; ${ }^{\dagger}$ tungbs@ims.vast.ac.vn
}

Received 18 September 2020

Accepted for publication 21 December 2020

Published 05 January 2020

\begin{abstract}
We proposed a multi-band metamaterial absorber (MA) based on high-order magnetic resonance using ring-shaped structures. We obtained three absorption peaks at $4.71 \mathrm{GHz}$ (the fundamental resonance), $13.39 \mathrm{GHz}$ (the third-order behavior), and $19.91 \mathrm{GHz}$ (the fifth-order behavior) with correspondent absorptions of 90\%, 100\%, and 99.33\%, respectively. Therefore, these high-order perfect absorptions are the potential to make the easier fabrication for the next generation of MAs, especially in high-frequency regions. Particular, the obtained results are further controlled using the LC circuit model. Besides, by scaling down the unit-cell size, the thirdand fifth-order absorptions are created in the $\mathrm{THz}$ frequency band.
\end{abstract}

Keywords: Metamaterials, Perfect absorber, High-order resonance.

Classification numbers: 81.05.Xj; 78.67.Pt. 


\section{INTRODUCTION}

During the last decades, metamaterials (MMs) have emerged as one of the most potential research field in the revolution of science and technology happening all over the world. Due to their extraordinary properties, MMs can be regarded in the top ten breakthrough topics changing modern science (ranked by the Materials Today journal [1]). The theoretical design of MMs was firstly proposed by Veselago in 1968 [2], for the simultaneous combination between the negative permeability $(\mu<0)$ and the negative permittivity $(\varepsilon<0)$ material. Thenceforth, in 1996 and 1999, J. B Pendry realized these media by using the wire and split-ring resonator (SRR) structure [3,4]. Particularly, Smith et al. demonstrated the existence of a measured negative refractive index (NRI) structure, constructed from wires and SRRs [5]. This success opened a new brand of artificial advanced materials- the so-call MMs. MMs are defined as artificially structured media in a size scale smaller than the wavelength of external stimulation. They are composed of periodically arranged unit cells, which play the role of "meta-atoms" in materials. Therefore, MMs can possess novel properties that cannot be easily attainable from natural materials. To date, there are many different research directions as the negative refractive index (NRI) [6-8], perfect absorption [9-11], invisible cloak [12], electromagnetically induced transparency [13,14] and so on. Among them, the perfect absorption feature is used for many potential applications, such as plasmonic sensors $[15,16]$, solar-energy capturing [17], and camouflage. The first metamaterial absorber (MA) has been demonstrated under the concept of MMs and a near-unity absorption peak can be realized at the resonant frequency [18]. To date, MAs have been demonstrated in every technologically relevant spectral range, from the microwave $[10,11,19]$, the $\mathrm{THz}[15,16]$, NIR [20] to the optical range [21]. However, most recent studies focus on the fundamental absorption mechanism of MM [9-11,22]. Moreover, one of the most interesting properties of MMs is that we can design and fabricate them working in the desired frequency range, from $\mathrm{GHz}$ to $\mathrm{THz}$ or even in the visible region. In recent years, THz-MAs have received the most research attention, which might be a solution to the shortage of $\mathrm{THz}$ sources and devices. However, at high frequencies, the small unit cell might be quite difficult for fabrication technologies. Research shows that the unit-cell size based on the high-order resonance increases significantly compared to that based on the first-order resonance. This can be further useful for practical manufacturing.

From this motivation, in this work, GHz- and THz-MAs are developed to create multi-band high-order absorption. Our investigations are attempt to clarified that multi-band absorption is simultaneously achieved by the fundamental-/third-/fifth-magnetic resonance in both the $\mathrm{GHz}$ and $\mathrm{THz}$ ranges. In addition, the influence of absorption on geometrical parameters is also estimated and theoretically explained by using the LC circuit model.

\section{SIMULATION}

In our simulation, the unit cell of the proposed MA is made of three sandwiched layers (copper ring - flame retardant FR-4 - copper continuous film), as shown in Fig. 1(a), by using the CST Microwave Studio software. The lossy-dielectric layer (dielectric constant of 4.3, losstangent of 0.025 ) is optimized by the thickness of $t_{s}=0.8 \mathrm{~mm}$. The thickness of both copper layers (electric conductivity of $5.96 \times 10^{7} \mathrm{~S} / \mathrm{m}$ ) is optimized to be $t_{m}=0.036 \mathrm{~mm}$. The geometrical parameters were set to be $a=18 \mathrm{~mm}, R_{1}=8.2 \mathrm{~mm}$ and $R_{2}=2 \mathrm{~mm}$. The direction of the incident wave $\mathbf{k}$ is parallel to the axis of the ring, while the $\mathbf{E}-\mathbf{H}$ plane is normal. The boundary conditions 
are set for periodic unit cells in the E-H plane. The absorption $A(\omega)$ is calculated as $A(\omega)=$ $1-R(\omega)-T(\omega)=1-\left|S_{11}\right|^{2}-\left|S_{21}\right|^{2}$, where the reflection $R(\omega)=\left|S_{11}\right|^{2}$, and the transmission $T(\omega)=\left|S_{21}\right|^{2}$, respectively. The equivalent circuit model LC of the structure is shown in Fig. 1 (b).

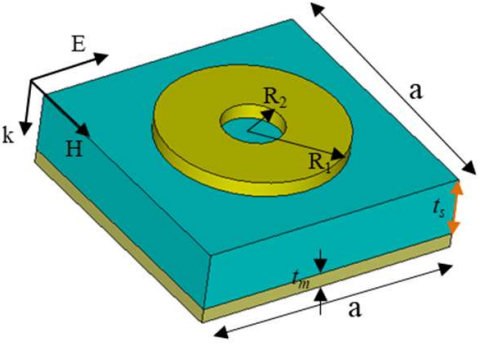

(a)

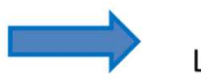

L

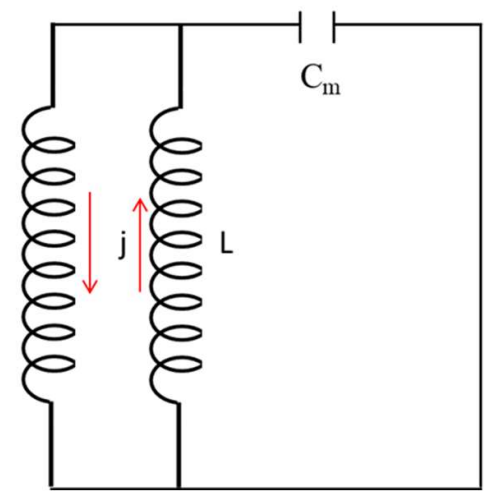

(b)

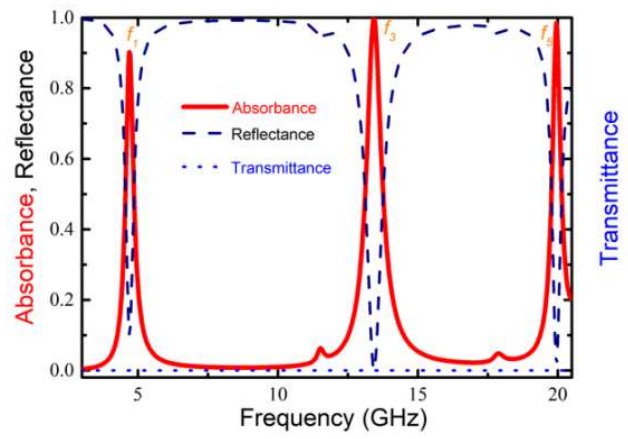

(c)

Fig. 1. (a) Unit cell of ring structure and (b) its equivalent circuit model at first-order magnetic resonance. (c) Simulated absorption, transmission and reflection.

\section{RESUTLS AND DISCUSSION}

\section{III.1. Absorption behavior at GHz frequencies}

In general, perfect absorption can be regarded as a difficult task in the optical region [21,23, 24], polarization dependence [18], and operation in a narrow frequency range [18,22] due to their complex structures for the recent fabrication techniques. To overcome the above disadvantages, we attempt to apply the basic ring-shaped structure because of high-symmetry property. From the theoretical point of view, unity absorption can be possibly achieved at a certain frequency by reducing reflection and transmission to zero simultaneously. The minimum reflection can be 
obtained by matching the impedance of the sample to the surrounding environment at the resonant frequency. Fig. 1(c) shows the simulated results of reflection, transmission and absorption in the frequency range from 2 to $21 \mathrm{GHz}$. We observed three absorption peaks at 4.71, 13.39, and 19.91 GHz with absorption of $90 \%, 100 \%$, and $99.33 \%$, respectively.
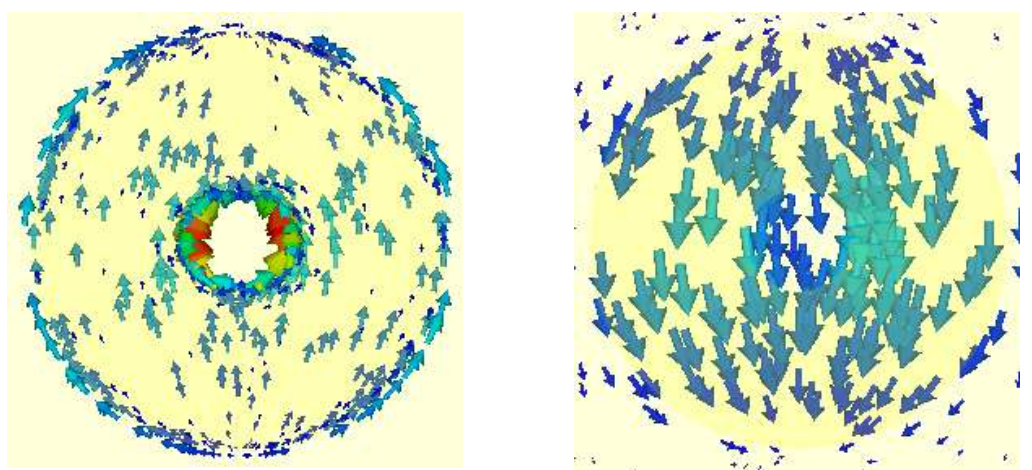

Front

(a) $f_{1}=4.71 \mathrm{GHz}$
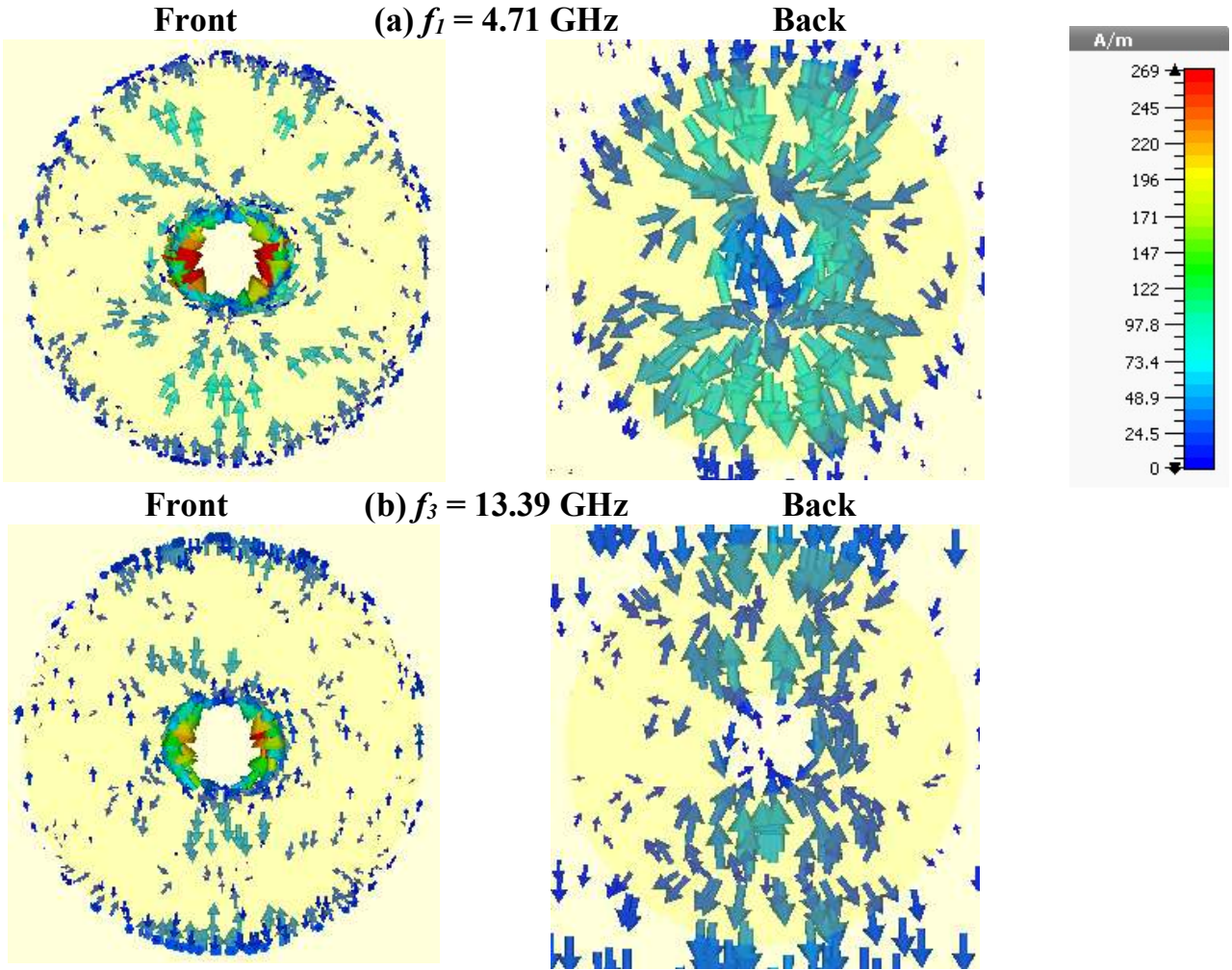

Front

(c) $f_{5}=19.91 \mathrm{GHz}$

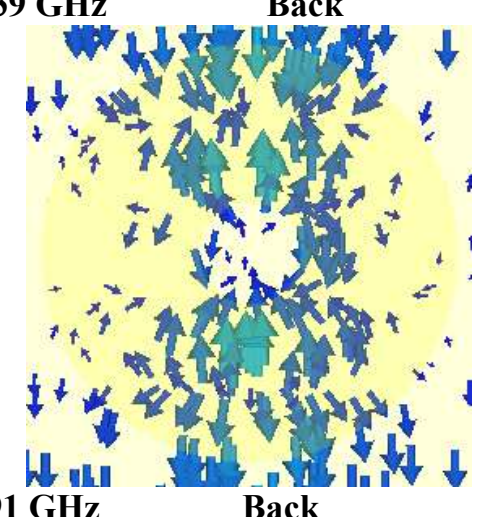

Fig. 2. Simulated induced surface current distributions of ring-shaped structure at (a) $f_{1}=4.71 \mathrm{GHz}$, (b) $f_{3}=13.39 \mathrm{GHz}$, and (c) $f_{5}=19.91 \mathrm{GHz}$.

As shown in Fig. 2(a), it is found that the surface-current distributions at $f_{1}=4.71$, $f_{3}=13.39$ and $f_{5}=19.91 \mathrm{GHz}$ confirm the anti-parallel currents on two metallic surfaces. This 
means that strong magnetic resonances are created at $4.71 \mathrm{GHz}$ (fundamental mode) $[25,26]$. However, from the current distribution at $13.39 \mathrm{GHz}$ and $19.91 \mathrm{GHz}$, (in Figs. 2 (b) and 2(c)), the surface currents are mainly located in the centers of three- and five-circular currents, but in different directions since the central circular current is opposite to the others, respectively. As a results, $f_{3}$ and $f_{5}$ are introduced to denote the third- and fifth-order magnetic resonances, respectively [25-27]. Thus, by using only the ring-structure, we created triple-band absorption with absorptions above $90 \%$ for $f_{1}$ and $f_{5}$, and absorption of $100 \%$ for $f_{3}$.

Building a formula of the absorption frequencies is extremely important to know how to manipulate absorption frequency effectively. In addition, the formula also benefits to the explanation of the deviation between simulated and measured spectra. In general, the dependence of the first- and higher-order absorption frequencies on the structural parameters is further verified by using the equivalent circuit model LC. According to Refs. [28, 29], the equivalent circuit diagram LC of ring structure at first-order magnetic resonance frequency as shown in Fig. 1 (b), in which the resistors are omitted for simplicity. In particular, the metal component on two metallic layers can be regarded as effective inductance $(L)$ in parallel, while the dielectric spacer acts like effective capacitance $C_{m}$.

\section{First- order magnetic resonance frequency}

In order to define the fundamental magnetic resonance depended on geometrical parameters, we calculated effective inductance and capacitance of the ring-shaped structure. Their distribution of the magnetic field density (B) at first-order magnetic resonance is shown in Fig. 3(a):

$$
B=\frac{\mu I}{w},
$$

where $\mu, I$ and $w$ are the free space permeability, the current on the ring pair and the width of the ring, respectively.

The magnetic flux was calculated by:

$$
\begin{aligned}
& \phi=\int \mathrm{BdS}, \\
& \phi=\int_{-\left(\mathrm{t}_{\mathrm{s}}+2 \mathrm{t}_{\mathrm{m}}\right) / 2}^{\left(\mathrm{t}_{\mathrm{s}}+2 \mathrm{t}_{\mathrm{m}}\right) / 2} \mathrm{dz} * 2 * \int_{\mathrm{R}_{2}}^{\mathrm{R}_{1}} \frac{\mu I}{2 \sqrt{R_{1}^{2}-y^{2}}} \mathrm{dy},
\end{aligned}
$$

where $N \phi=L I$ and $\mathrm{N}=1$ is denoted for the number of turns and the inductance of the ring-pair system, respectively. The inductance $L$ can be approximated as:

$$
\begin{aligned}
L & =\int_{-\left(t_{s}+2 t_{m}\right) / 2}^{\left(t_{s}+2 t_{m}\right) / 2} \mathrm{dz} \int_{-\left(\mathrm{R}_{1}-\mathrm{R}_{2}\right)}^{\left(\mathrm{R}_{1}-\mathrm{R}_{2}\right)} \frac{\mu}{2 \sqrt{R_{1}^{2}-y^{2}}} \mathrm{dy}, \\
L & =\mu\left(t_{s}+2 t_{m}\right)\left[\frac{\pi}{2}-\arcsin \left(\frac{R_{2}}{R_{1}}\right)\right], \\
L_{m} & =\frac{L}{2}=\frac{\mu\left(t_{s}+2 t_{m}\right)\left[\frac{\pi}{2}-\arcsin \left(\frac{R_{2}}{R_{1}}\right)\right]}{2} .
\end{aligned}
$$

On the other hand, when the electromagnetic (EM) wave impinges on the material, the Lorentz force, which is caused by the magnetic field, pushes the charge on both ends of the ring. 

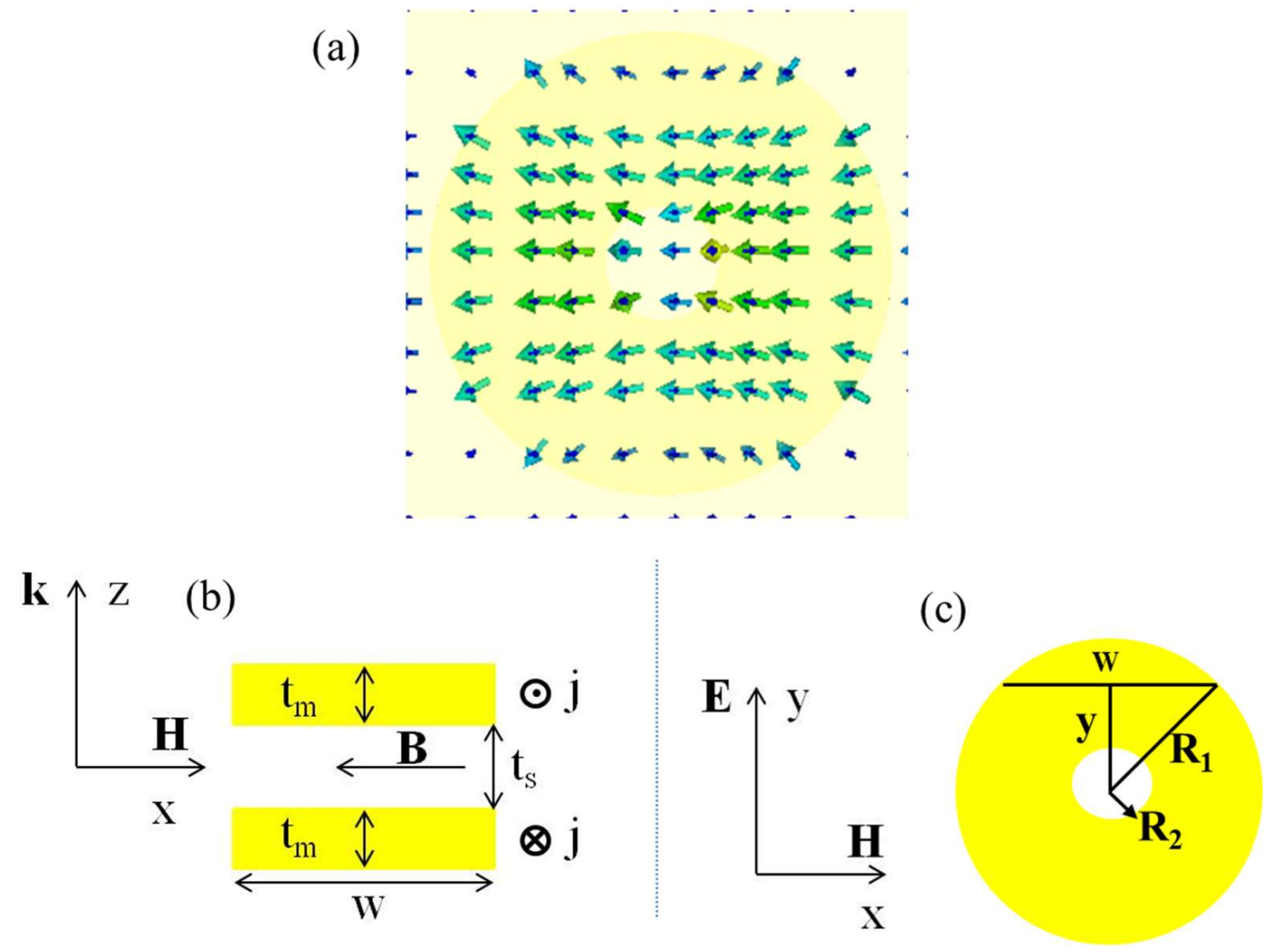

Fig. 3. (a) Distribution of the magnetic field density of the ring-shaped structure at the first-order magnetic resonance, (b) View of ring-structure on (left) (k, H) plane and and (c) (right) (E, H) plane.

Then, the charge of the ring focuses only on the two ends of the ring, forming two flat capacitors. The capacitance value of the capacitors may be determined by the equation:

$$
C_{m}=\varepsilon \frac{\pi c_{1}\left(R_{1}^{2}-R_{2}^{2}\right)}{t_{s}}
$$

where $C_{1}$ is a geometrical factor $0.2 \leq c_{1} \leq 0.3$ [30] and $\varepsilon$ is the permittivity of FR-4. Consequently, we can obtain the magnetic resonance frequency as

$$
\begin{aligned}
\omega & =\frac{1}{\sqrt{L_{m} C_{m}}}=\frac{\sqrt{2 t_{s}}}{\sqrt{\pi c_{1} \varepsilon \mu\left(t_{s}+2 t_{m}\right)\left(R_{1}^{2}-R_{2}^{2}\right)\left(\frac{\pi}{2}-\arcsin \left(\frac{R_{2}}{R_{1}}\right)\right)}} . \\
f_{m 1} & =\frac{1}{2 \pi \sqrt{L_{m} C_{m}}}=\frac{\sqrt{2 t_{s}}}{2 \pi \sqrt{\pi c_{1} \varepsilon \mu\left(t_{s}+2 t_{m}\right)\left(R_{1}{ }^{2}-R_{2}{ }^{2}\right)\left(\frac{\pi}{2}-\arcsin \left(\frac{R_{2}}{R_{1}}\right)\right)}} .
\end{aligned}
$$


If $R_{2}<<R_{1}$, the ring shape will transform into a disk shape and the magnetic resonance frequency is approximately as:

$$
\begin{aligned}
\omega & =\frac{1}{\sqrt{L_{m} C_{m}}}=\frac{2 \sqrt{t_{s}}}{\pi R_{1} \sqrt{c_{1} \varepsilon \mu\left(t_{s}+2 t_{m}\right)}} . \\
f_{m 1} & =\frac{1}{2 \pi \sqrt{L_{m} C_{m}}}=\frac{\sqrt{t_{s}}}{\pi^{2} R_{1} \sqrt{c_{1} \varepsilon \mu\left(t_{s}+2 t_{m}\right)}} .
\end{aligned}
$$

\section{Third-order magnetic resonance frequency}

In Fig. 2(b), the current distribution at the third-order resonant frequency $\left(f_{3}=13.39 \mathrm{GHz}\right)$, there are three pairs of anti-parallel surface current and alternate directions. Therefore, the nature of the resonance at this frequency is magnetic resonance $[25,26]$. The electric field distribution shows that the electric field focuses on four positions of the structure, as plotted in Fig. 4(a).

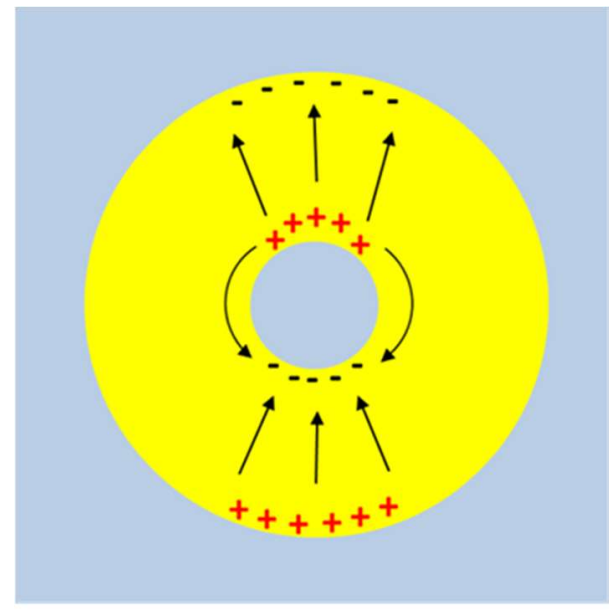

(a)

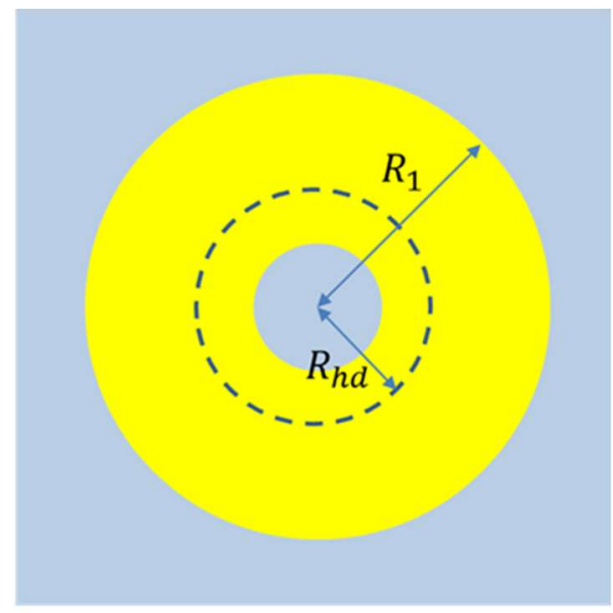

(b)

Fig. 4. (a) Distributions of induced charges and surface current at $13.39 \mathrm{GHz}$ (b) Effective radius at third-order resonance frequency, which has properties as the first-order resonance frequency.

From Fig. 4(b), a specific area corresponding to the effective radius $R_{h d}$ is considered. In this effective area, it can be noted that the induced surface current distributions are similar in comparison with the case of first-order magnetic resonance. Thus, to calculate the third-order resonance, instead of studying all of the structure, the radius $R_{1}$ is equivalently transformed to the effective radius.

The effective radius value will be taken approximately by $R_{h \mathrm{~d}} \approx \frac{R_{1}}{2}$. By using $R_{h d}$ instead of $R_{1}$, the third-order magnetic resonance frequency can be estimated as:

$$
f_{m 3}=\frac{1}{2 \pi \sqrt{L_{m} C_{m}}}=\frac{\sqrt{2 t_{s}}}{2 \pi \sqrt{\pi c_{2} \varepsilon \mu\left(t_{s}+2 t_{m}\right)\left(R_{h d}{ }^{2}-R_{2}^{2}\right)\left(\frac{\pi}{2}-\arcsin \left(\frac{R_{2}}{R_{h d}}\right)\right)}} .
$$


In Eq. (9), the total charge of the ring focuses only on two regions. However, in Eq. (12), which determines the third-order resonant frequency, the charges are concentrated in four areas (as shown in Fig. 4). Substituting the structural parameters $\left(R_{1}=8.2 \mathrm{~mm}, R_{2}=2 \mathrm{~mm}, t_{d}=0.8 \mathrm{~mm}\right.$, $\left.t_{s}=0.036 \mathrm{~mm}, \varepsilon=4.3, C_{1}=0.2\right)$ into Eq. (9), $f_{m 1}$ was obtained to be $4.36 \mathrm{GHz}$ and into Eq. (12), $f_{m 3}$ was obtained to be $14.5 \mathrm{GHz}$. The deviation between simulated and calculated results for $f_{3}$ can be explained because $R_{h d}$ and $C_{2}$ have been approximately applied.
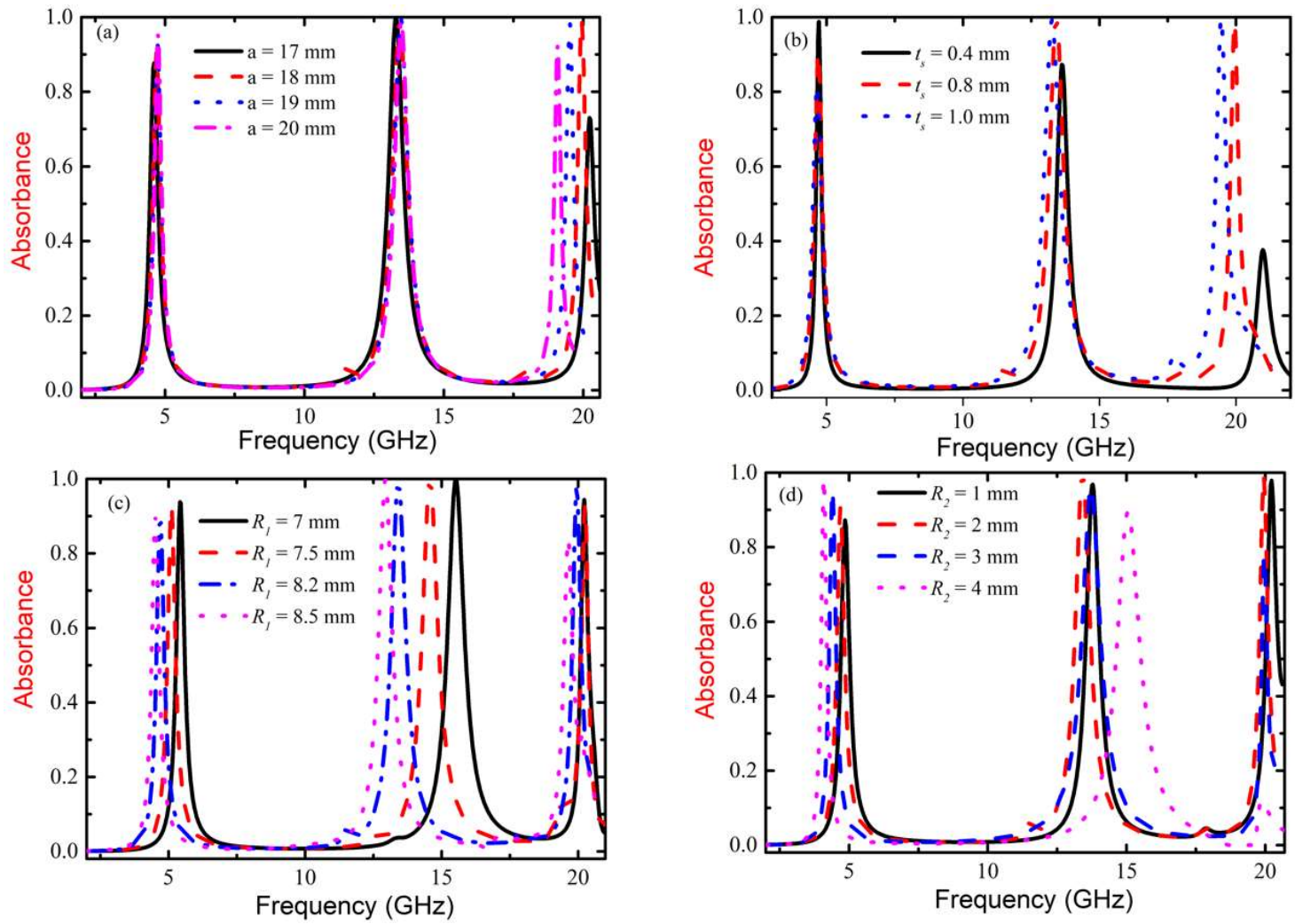

Fig. 5. Influence of (a) lattice constant $a$, (b) dielectric thickness $t_{d}$, (c) radius $R_{1}$, (d) radius $R_{2}$ on absorption spectrum.

Understanding the dependence of resonance on structural parameters are very necessary to optimize the structure. Figure 5 shows the dependence of simulated absorption on the structural parameters. It can be noticed that the absorption frequencies at first- and third-order resonances are strongly influenced by the parameters $R_{1}$ and $R_{2}$, but not by the lattice constant $a$ or the dielectric thickness $t_{s}$. The dependence of the resonant frequency on the structural parameters is perfectly matched by Eqs. (9) and (12). Fig. 5 presents the dependence of the triple-band absorption $\left(f_{1}, f_{3}\right.$ and $f_{5}$ ) on structural parameters, which are optimized to be $a=18 \mathrm{~mm}, t_{s}=0.8 \mathrm{~mm}, R_{1}=8.2 \mathrm{~mm}$, and $R_{2}=2.0 \mathrm{~mm}$.

Recently, most MAs have been proposed based on conventional structures such as SRRs and cut-wires. Therefore, their absorption feature is strongly depended on the polarization of EM 
wave, which limits practical applications in some areas. This drawback can be overcome by taking advantage of the proposed ring-shaped structure. The independent absorption on the polarization of the EM wave is confirmed in Fig. 6 (a). The simulation shows that the absorption magnitudes at $f_{1}, f_{3}$ and $f_{5}$ are unchanged as the polarization angle is increased from 0 to $90^{\circ}$. Besides, the impacts of incident angles on the absorption is also shown in Fig. 6(b). From Fig. 6(b), the absorption responses a low sensitivity to the incident angles between $0^{\circ}$ and $20^{\circ}$. The absorption magnitudes at $f_{1}$ and $f_{3}$ are unchanged as the incident angles is increased from $0^{\circ}$ to $60^{\circ}$. However, the absorption magnitudes at $f_{5}$ changed as the incident angles is increased from $40^{\circ}$ and appears some new absorption peaks. The appearance of the new peaks can be explained that increasing the incident angles will change the effective impedance of the front and back sides (creating an asymmetric structure) [31, 32].
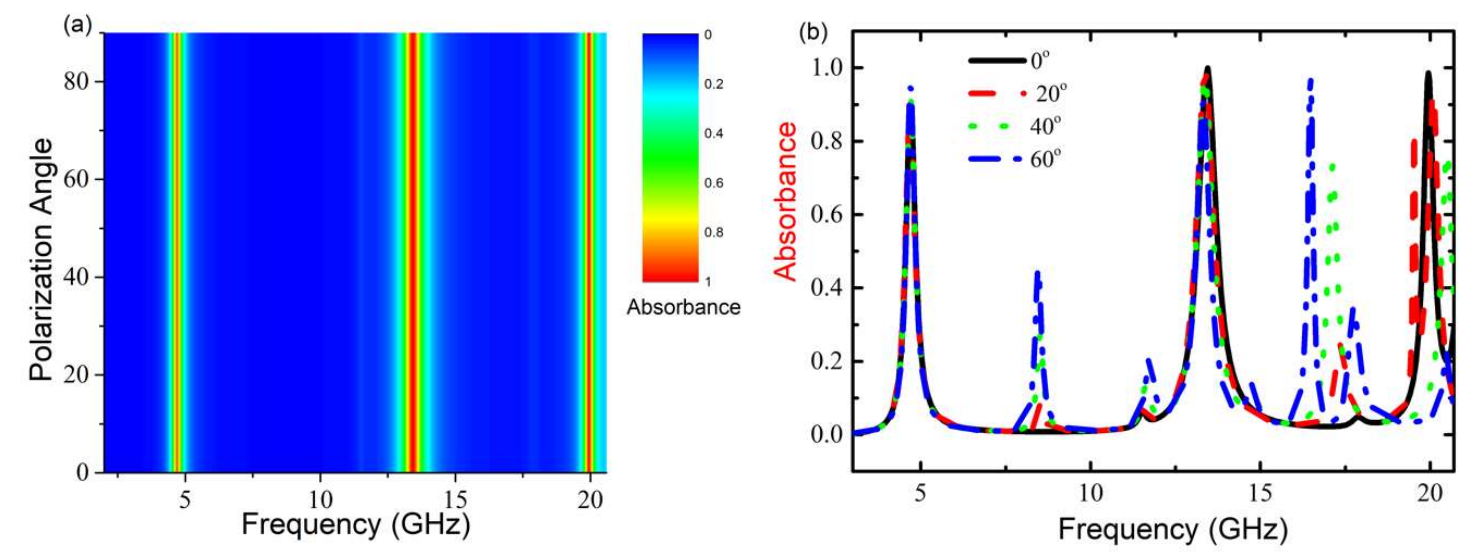

Fig. 6. Absorption spectra according to (a) polarization angles and (b) incident angles.

\section{III.2. Absorption behavior at THz frequencies}

The exploitation of the ring-structure to multiband absorber is investigated in the $\mathrm{THz}$ regime, as shown in Fig. 7. By reducing the structural parameter values by scale factors of 0.001 , the resonance frequency of MMs can be increased from $\mathrm{GHz}$ to $\mathrm{THz}$ bands. It is noted that we fix the dielectric loss tangent to 0.025 in order to keep the nature of MA unchanged. Figure 7 shows that three absorption peaks are activated at $f_{1}^{\prime}=4.71, f_{3}^{\prime}=13.39$ and $f_{5}^{\prime}=19.91 \mathrm{THz}$ with absorption of $98 \%, 90 \%$, and $80 \%$, respectively. This presence of multiple absorption peaks opens the possibility for electronic devices using multiband metamaterial absorbers not only in the $\mathrm{GHz}$ but also in the THz range. The surface-current distributions of the ring-shaped structure at $f_{1}^{\prime}, f_{3}^{\prime}$ and $f_{5}^{\prime}$ in Fig. 8 completely similar in Fig. 2. This demonstrates that these resonances are also due to the first-, third-, and fifth-order magnetic resonances (see the anti-parallel surface currents at all absorption peaks). However, it can be realized that the magnitudes of absorption at $f_{3}^{\prime}$ and $f_{5}^{\prime}$ are significantly reduced. This phenomenon can be explained through the contributed role of the Ohmic loss in the higher frequency regime, leading to a remarkable decrease in the magnitude of absorption in the higher frequency regime. 
MULTIBAND METAMATERIAL ABSORBER BASE ON HIGH-ORDER MAGNETIC RESONANCE ...

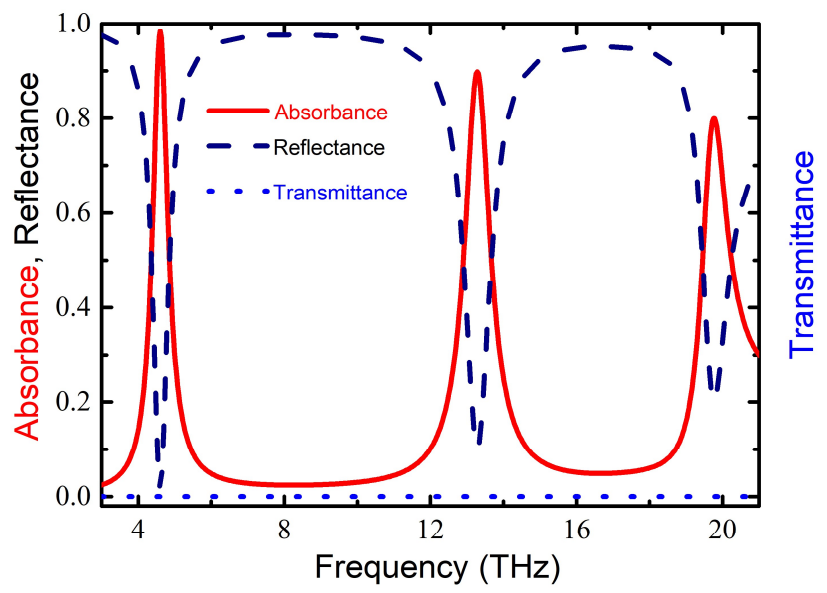

Fig. 7. Simulated absorption spectrum of the single ring-shaped structure at $\mathrm{THz}$ frequency absorption spectrum.

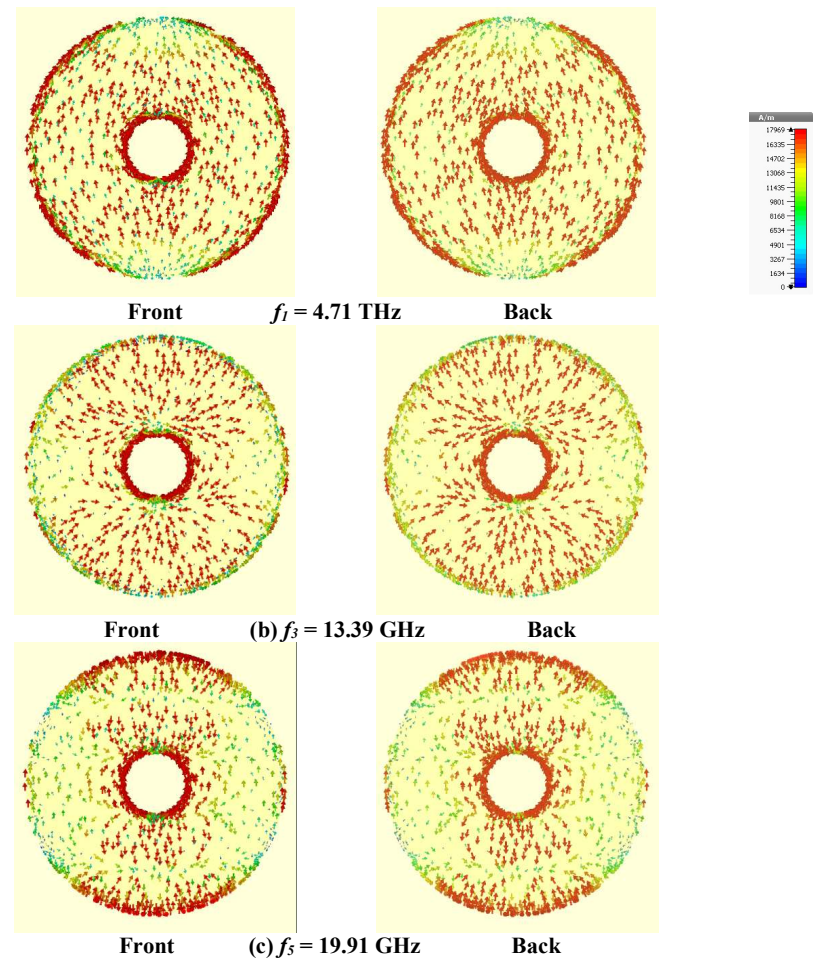

Fig. 8. Numerical surface current distributions of ring-shaped structure at (a) $f_{1}=$ $4.71 \mathrm{THz}$, (b) $f_{3}=13.39 \mathrm{THz}$, (b) $f_{5}=19.91 \mathrm{THz}$. 


\section{CONCLUSION}

We numerically investigated the absorption feature of a simple MA in the state of high-order magnetic resonances. By using the ring-shaped structure triple-band absorption is obtained at $4.71,13.39$, and $19.91 \mathrm{GHz}$, corresponding to the fundamental mode, the third- and the fifth-order resonant frequencies, with absorption of $90 \%, 100 \%$, and $99.33 \%$, respectively. In addition, the obtained results can be predicted theoretically by exploiting the LC-circuit model. Furthermore, by scaling down the unit-cell size, these high-order perfect absorption behaviors are also effectively well maintained in the $\mathrm{THz}$ band. This strong advantage confirms that we can challenge for highfrequency applications using a larger-size sample, which is fabricated in easier and cheaper way in reality.

\section{ACKNOWLEDGMENT}

This research was funded by Vietnam National Foundation for Science and Technology Development (NAFOSTED) under Grant No. "103.99-2018.35" and by Vietnam Academy of Science and Technology, under Grant No. NCVCC42.03/20-20.

\section{REFERENCES}

[1] J. Wood, Mater. Today 11 (2008) 40.

[2] V. G. Veselago, Sov. Phys. Usp. 10 (1968) 509.

[3] J. B. Pendry, A. J. Holden, W. J. Steward and I. Youngs, Phys. Rev. Lett. 76 (1996) 4773.

[4] J. B. Pendry, A. J. Holden, D. J. Robbins, and W. J. Stewart, IEEE Trans. Microwave Theory Tech. 47 (1999) 2075.

[5] D. R. Smith, W. J. Padilla, D. C. Vier, S. C. Nemat-Nasser, and S. Schultz, Phys. Rev. Lett. 84 (2000) 4184.

[6] N. T. Hien, B. S. Tung, Y. Sen, A. E. V. Guy, P. Lievens, V. D. Lam and J. Ewald, App. Phys. Lett. 109 (2016) 221902.

[7] N. T. Hien, L. D. Hai, B. S. Tung, B. X. Khuyen, N. X. Ca, V. D. Lam, J. Phys. D: Appl. Phys. 53 (2020) 175501.

[8] T. Shuaishuai, R. Chunyu and T. Weipeng, Appl. Phys. Lett. 114 (2019) 204101.

[9] P. Kevin, K. Matthias, B. Julian, B. Andre, A. Philipp, K. Ulrich and R. Stefan, Nature 567 (2019) 351.

[10] G. Singh, H. Sheokand, K. Chaudhary, K. V. Srivastava, J. Ramkumar and S. A. Ramakrishna, J. Phys. D: Appl. Phys. 52 (2019) 385304.

[11] B. X. Khuyen, B. S. Tung, N. T. Tung, N. T. Hien, Y. J. Kim, L. Y. Chen, Y. P. Lee, P. T. Linh and V. D. Lam, J. Phys. D: Appl. Phys. 53 (2020) 105502.

[12] J. B. Pendry, D. Schurig, D. R. Smith, Science 312 (2006) 1780.

[13] J. S. Hwang, B. S. Tung and Y. P. Lee, J. Korean Phys. Soc. 72 (2018) 1491.

[14] R. Kun, H. Yumeng, R. Xiaobin, Z. Ying, H. Qun, W. Liedong and X. Maojun, J. Phys. D: Appl. Phys. 53 (2020) 122593.

[15] N. Liu, M. Mesch, T. Weiss, M. Hentschel, H. Giessen, Nano Lett. 10 (2010) 2342.

[16] F. B. P. Niesler, J. K. Gansel, S. Fischbach, M. Wegener, Appl. Phys. Lett. 100 (2012) 203508.

[17] K. Aydin, V. E. Ferry, R. M. Briggs, H. A. Atwater, Nat. Commun. 2 (2011) 517.

[18] N. I. Landy, S. Sajuyigbe, J. J. Mock, D. R. Smith, W. J. Padilla, Phys. Rev. Lett. 100 (2008) 207402.

[19] T. M. Cuong, P. V. Hai, H. T. Hung, N. T. Thuy, D. H. Tung, B. X. Khuyen, B. S. Tung, L. D. Tuyen, P. T. Linh, V. D. Lam, Sci. Rep. 10 (2020) 1810.

[20] C. Lii, J. Qiu, J. Y. Ou, Q. H. Liu, J. Zhu, ACS Appl. Nano Mater. 2 (2019) 3231.

[21] H. Zhang, L. Feng, Y. Liang and T. Xu, Nanoscale 11 (2019) 437.

[22] D. T. Anh, D. T. Viet, P. T. Trang, N. M. Thang, H. Q. Quy, N. V. Hieu, V. D. Lam and N. T. Tung, AIP Advances 5 (2015) 007119.

[23] H. Tao, C. M. Bingham, A. C. Strikwerda, D. Pilon, D. Shrekenhamer, N. I. Landy, K. Fan, X. Zhang, W. J. Padilla and R. D. Averitt, Phys. Rev. B 78 (2008) 241103. 
[24] J. Grant, Y. Ma, S. Saha, A. Khalid and D. R. Cumming, Opt. Lett. 36 (2011) 3476.

[25] N. T. Tung, V. T. T. Thuy, J. W. Park, J. Y. Rhee, and Y. P. Lee, Jour. Appl. Phys. 107 (2010) 023530.

[26] D. T. Viet, N. T. Tung, N. T. Hien, Y. P. Lee, B. S. Tung and V. D. Lam, J. Nonlinear Opt. Phys. Mater. 21 (2012) 1250019

[27] S. Jung, Y. Kim, Y. J. Yoo, J. S. Hwang, B. X. Khuyen, L. Chen and Y. Lee, J. Electron. Mater. 49 (2020) 1677.

[28] L. Zhu, F. Meng, J. Fu, Q. Wu and J. Hua, Opt. Express 20 (2012) 4494.

[29] L. Solymar and E. Shamonina, Waves in metamaterials, Oxford University, 2009.

[30] J. Zhou, E. N. Economon, T. Koschny and C. M. Soukoulis, Opt. Lett. 31 (2006) 3620.

[31] D. H. Tiep, B. S. Tung, B. X. Khuyen and V. D. Lam, Vietnam J. Sci. Technol. 57 (2019) 477.

[32] L. D. Hai, V. D. Qui, D. H. Tiep, P. Hai, T. T. Giang, T. M. Cuong, B. S. Tung and V. D. Lam, J. Electron. Mater 46 (2017) 3757. 